Pacific Journal of Mathematics

IMAGE" OF CONVEX SURFACES IN LOCALLY CONVEX
SPACES

THEODORE WILLIS LAETSCH 


\title{
NORMAL CONES, BARRIER CONES, AND THE "SPHERICAL IMAGE" OF CONVEX SURFACES IN LOCALLY CONVEX SPACES
}

\author{
THEODORE LAETSCH
}

\begin{abstract}
This paper discusses relations between a closed, convex set in a locally convex space and its normal cones and barrier cone in the dual space. Results of $W u$ and de Andrade on the Gauss map and spherical image of a convex hypersurface in a Hilbert space are generalized to the topological vector space situation, and additional information is obtained on the relation of the interior of the spherical image and barrier cone to the size and shape of the given convex set.
\end{abstract}

1. Introduction. Suppose $K$ is a closed, convex set with a smooth boundary $M$ and a nonempty interior in a finite- or infinitedimensional inner product space. Let $\Sigma$ be the unit sphere of the space. The "Gauss map" $\gamma: M \rightarrow \Sigma$ associates to each point $x_{0} \in M$ the outer unit normal vector, $\gamma\left(x_{0}\right)$, to $M$ at $x_{0}$. The outer unit normal can be characterized as the unit vector $u=\gamma\left(x_{0}\right)$ which is uniquely determined (when $M$ is smooth) by the condition

$$
\left\langle x-x_{0}, u\right\rangle \leqq 0 \text { for all } x \in K \text {. }
$$

If $M$ is not smooth, it is customary to call all (nonzero) vectors $u$ satisfying (1.1) "normal" to $M$ at $x_{0}$, and the Gauss map becomes a multivalued function associating to each $x_{0} \in M$ the set $\gamma\left(x_{0}\right)$ of all unit vectors satisfying (1.1). The "spherical image" of $M$ (or of $K$ ) is $\gamma(M)$, the image in $\Sigma$ of $M$ under $\gamma$.

In a number of recent papers, [31, 9, 30, 1, 2], several authors have studied properties of the spherical image and the relation of these to properties of $M$. Wu $[31,30]$ has treated the finite dimensional case and de Andrade [1,2] considered the Hilbert space case.

It is the purpose of this note to point out the relation between these results - especially those of de Andrade - and certain fundamental theorems in the general theory of convex sets and functions in topological vector spaces: In particular, the Bishop-Phelps theorem, the theorems of James on weak-compactness and reflexivity, and the theorems of Moreau and Rockafellar on the relation between continuity properties of a convex functional and the size of the level sets of its convex conjugate function. With the help of these general theorems, we are able to obtain very simple proofs, not only of the 
theorems of $\mathrm{Wu}$ and de Andrade, but of the natural generalizations of these theorems to Banach spaces and locally convex topological vector spaces.

The most significant geometric distinction between the finite and infinite dimensional cases is that, in the finite-dimensional case, if $K$ is unbounded, it contains a half-line; in the infinite-dimensional case, there always exists an unbounded $K$ which contains no half-line. Section 3 below is devoted primarily to generalizations of de Andrade's characterization [2] of the three possibilities ( $M$ bounded, $M$ unbounded but bounds no half-line, $M$ bounds a half-line) in terms of the spherical image of $M$. In $\S 5$, we carry the analysis further by noting relationships between the interior of the spherical image of $M$ and properties of the intersection of $M$ and $K$ with hyperplanes and halfspaces. We incidentally obtain the generalization (see Theorem 3.1.2 and Corollary 5.2.4) of the result of $\mathrm{Wu}$ [30] and de Andrade [1] that the interior and the closure (relative to the unit sphere $\Sigma$ ) of the spherical image are convex. (Wu gives a threedimensional example in which the spherical image itself is not convex.) The proofs of the results of $\S \S 3$ and 5 are given in $\S \S 4$ and 6 respectively; these proofs usually consist of pointing out the applicability of the relevant general theorem on convex functions.

Throughout, the transition from the functional analytic statement to the geometric statement is based on the elementary observation that the spherical image can be identified with the normalized set of all linear functionals which attain their supremum on $K$.

\section{Notation and terminology.}

2.1. Let $E$ and $E^{\prime}$ be two real linear spaces which are placed in duality by a bilinear form $\left\langle x, x^{\prime}\right\rangle\left(x \in E, x^{\prime} \in E^{\prime}\right)$ which separates points of $E$ and of $E^{\prime}$ [7, Chap. IV; 17, Chap. 5]. We assume, whenever a topology is needed but not specifically described, that $E$ or $E^{\prime}$ is provided with a topology compatible with the duality (so that $E^{\prime}$ or $E$ may be identified with the dual space of $E$ or $E^{\prime}$, respectively). The weakest and strongest such topologies (on $E$, for example) are the weak topology $\sigma\left(E, E^{\prime}\right)$ and the Mackey topology $\tau\left(E, E^{\prime}\right)$. We will also refer to the strong topology, $\beta\left(E^{\prime}, E\right)$, on $E^{\prime}$. When $E$ is a Banach space, the $\beta\left(E^{\prime}, E\right)$ topology is the same as the norm topology on the dual $E^{\prime}$; it is not, in general, compatible with the duality. When the topology $\beta\left(E^{\prime}, E\right)$ on the dual of a locally convex space $E$ is compatible with the duality of $E$ and $E^{\prime}$, then $E$ is called semireflexive. A Banach space is semireflexive if and only if it is reflexive.

For $S \subseteq E, \operatorname{cl}(S)$ and int $(S)$ denote the closure and interior of 
$S$, respectively, relative to the topology in question. In a Banach space, these symbols unmodified always refer to the norm topology.

2.2. We recall some terminology from the theory of convex sets and convex functions (cf. $[11 ; 12 ; 26 ; 27])$. Let $\left(E, E^{\prime}\right)$ be a dual pair as described above, and let $K$ be a closed, convex subset of $E$. An internal point of $K$ is a point $x \in K$ such that every half-line in $E$ emanating from $x$ contains a point of $K$ distinct from $x$; this is sometimes described by saying that $K$ is radial or absorbing at $x$. The set of all internal points of $K$ is the core of $K$, denoted by $\operatorname{cor}(K)$. We define bd $(K)=K \backslash \operatorname{cor}(K)$. The recession cone (or asymptotic cone) of $K$, denoted by $0^{+} K$, is the set of all $y \in E$ such that, for every $x \in K$, the half-line $x+\lambda y(\lambda \geqq 0)$ belongs to $K ; 0^{+} K$ is a closed, convex cone which always contains at least the 0 of $E$, and it can be alternatively characterized by

$$
0^{+} K=\{y \in E: K+y \subseteq K\}
$$

[29;27, §8]. By definition, $0^{+} K \neq\{0\}$ if and only if $K$ contains a halfline. The indicator function $\delta_{K}$ of $K$ is the extended real-valued function which is zero on $K$ and $+\infty$ on $E \backslash K$; this function is convex (since $K$ is) and lower semi-continuous (since $K$ is closed). We say that $K$ is a convex body if the interior of $K$ is not empty (with this definition, a convex body is not necessarily bounded).

The barrier cone of $K$, denoted by bar $(K)$, is the set of all elements of $E^{\prime}$ which, when considered as continuous linear functionals on $E$, are bounded above on $K$; it is a convex cone in $E^{\prime}$ containing the 0 of $E^{\prime}$. Clearly, $K$ is bounded if and only if bar $(K)=E^{\prime}$. The normal cone to $K$ at $x_{0}\left(x_{0} \in K\right)$ is the set of $x^{\prime} \in E^{\prime}$ such that $\left\langle x-x_{0}, x^{\prime}\right\rangle \leqq 0$ for all $x \in K$; it coincides with the subdifferential of $\delta_{K}$ at $x_{0}$, that is, the set

$$
\partial \delta_{K}\left(x_{0}\right)=\left\{x^{\prime} \in E^{\prime}: \text { for all } x \in E, \delta_{K}(x) \geqq \delta_{K}\left(x_{0}\right)+\left\langle x-x_{0}, x^{\prime}\right\rangle\right\},
$$

and hence we denote it by $\partial \delta_{K}\left(x_{0}\right)$ (cf. [11, $\left.\S 9\right]$ ). For $x_{0} \notin K, \partial \delta_{K}\left(x_{0}\right)$ is empty; for $x_{0} \in K, \partial \delta_{K}\left(x_{0}\right)$ is a $\sigma\left(E^{\prime}, E\right)$-closed, convex cone in $E^{\prime}$ containing 0 , and $\partial \delta_{K}\left(x_{0}\right)=\{0\}$ for every $x_{0} \in \operatorname{cor}(K)$. For $x_{0} \in \mathrm{bd}(K)$, the nonzero elements (if any) of $\partial \delta_{K}\left(x_{0}\right)$ are precisely the supporting functionals of $K$ at $x_{0}$, that is, the nonzero $x^{\prime} \in E^{\prime}$ satisfying

$$
\left\langle x_{0}, x^{\prime}\right\rangle=\sup \left\{\left\langle x, x^{\prime}\right\rangle: x \in K\right\} \text {, }
$$

and $x_{0}$ is a support point of $K$ if and only if $\partial \delta_{K}\left(x_{0}\right)$ contains nonzero elements. The set of all supporting functionals of $K$, together with the zero functional, is thus 


$$
\partial \delta_{K}(K)=\partial \delta_{K}(\mathrm{bd}(K))=\bigcup_{x \in K} \partial \delta_{K}(x) .
$$

Clearly, $\partial \delta_{K}(K) \subseteq \operatorname{bar}(K)$.

The polar (in $E^{\prime}$ ) of $K \subseteq E$ is

$$
K^{\circ}=\left\{x^{\prime} \in E^{\prime}:\left\langle x, x^{\prime}\right\rangle \leqq 1 \text { for all } x \in K\right\} \text {. }
$$

Analogously, the polar (in $E$ ) of $C \subseteq E^{\prime}$ is

$$
{ }^{\circ} C=\left\{x \in E:\left\langle x, x^{\prime}\right\rangle \leqq 1 \text { for all } x^{\prime} \in C\right\} .
$$

If $C$ is a convex cone in $E^{\prime}$, this is equivalent to

$$
{ }^{\circ} C=\left\{x \in E:\left\langle x, x^{\prime}\right\rangle \leqq 0 \text { for all } x^{\prime} \in C\right\} .
$$

The basis for much of what follows is the known fact that ${ }^{\circ}[\operatorname{bar}(K)]=0^{+} K[29$, Theorem $2 \mathrm{~A}(\mathrm{~d})]$; that is, $y \in 0^{+} K$ if and only if $\left\langle y, x^{\prime}\right\rangle \leqq 0$ for all $x^{\prime} \in \operatorname{bar}(K)$. (De Andrade's Lemma $1[1,2]$ is a Hilbert space version of this result.)

2.3. To relate the above notation with that used by de Andrade [2], we assume that $E$ is a Banach space, let $\Sigma^{\prime}$ be the boundary of the unit ball in the dual space $E^{\prime}$, set $M=\mathrm{bd}(K)$, and define a multi-valued map $\nu: M \rightarrow \Sigma^{\prime}$ by

$$
\begin{aligned}
\nu(x) & =\left\{x^{\prime} \in E^{\prime}: x^{\prime} \in \Sigma^{\prime} \text { and }-x^{\prime} \in \partial \delta_{K}(x)\right\} \\
& =\Sigma^{\prime} \cap\left[-\partial \delta_{K}(x)\right] .
\end{aligned}
$$

If $K$ is a convex body, then $M$ is precisely the topological boundary of $K$ and $\nu(x)$ is nonempty for every $x \in M$ (support theorem for convex bodies [12, page 64]). If, furthermore, $M$ is smooth, then $\nu(x)$ is the normalized linear functional which determines the unique supporting hyperplane to $K$ at $x$, and if $E$ is Hilbert space, the direction of $\nu(x)$ is normal to this hyperplane and points from $x$ towards the interior of $K$.

Since $\partial \delta_{K}(M)$ is a cone in $E^{\prime}$ (it contains all positive multiples of its elements) and $\nu(M)=\Sigma^{\prime} \cap\left[-\partial \delta_{K}(M)\right]$, the topological properties of $\partial \delta_{K}(M) \backslash\{0\}$ relative to $E^{\prime}$ are precisely analogous to the properties of $\nu(M)$ relative to $\Sigma^{\prime}$. Furthermore, in a Hilbert space, a cone $C$ which is not a line is convex if and only if $C \cap \Sigma^{\prime}$ is geodesically convex. Thus, unless $M$ consists of two parallel halfplanes, the convexity properties of $\partial \delta_{K}(M)$ and its closure are precisely analogous to the geodesic-convexity properties of $\nu(M)$. (Cf. [4, Theorem 28.13].)

3. Relations between $K$, bar $(K)$ and $\partial \delta_{K}[\mathrm{bd}(K)]$.

3.1. de Andrade's result [2, Theorem A] can be formulated as 
a correspondence between properties of the boundary $M$ of a convex body $K$ in a Hilbert space and properties of $\partial \delta_{K}(M)$ :

$M$ is bounded if and only if $\partial \delta_{K}(M)$ is the entire space; $M$ is unbounded and bounds no half-line (i.e., $0^{+} K=\{0\}$ ) if and only if $\partial \delta_{K}(M)$ is dense and has empty interior; $M$ is unbounded and bounds a half-line if and only if $\partial \delta_{K}(M)$ is contained in a halfspace.

This result continues to be valid precisely as stated in any reflexive Banach space (cf. Theorem 3.1.3 and its corollary below), and it follows from James criterion for reflexivity that it is valid only in reflexive spaces (when $K$ has nonempty interior; Theorem 3.1.5 below). Now in a reflexive space, the weak compactness of a bounded $K$ implies $\partial \delta_{K}(M)=$ bar $(K)$; and, more generally, in a Banach space, $\partial \delta_{K}(M)$ is dense in bar $(K)$ (Bishop-Phelps theorem, see below). Hence the content of the above assertion is essentially unchanged by replacing $\partial \delta_{K}(M)$ by bar $(K)$, and in this form we obtain the broadest and simplest generalization (all proofs are given in the next section):

3.1.1. THEOREM. Let $\left(E, E^{\prime}\right)$ be a dual system of vector spaces with topologies compatible with the duality. Let $K$ be a nonempty, closed, convex subset of $E$, and set $M=\mathrm{bd}(K)$. Then

(a) $K$ is bounded if and only if $\operatorname{bar}(K)=E^{\prime}$.

(b) $0^{+} K \neq\{0\}$ if and only if bar $(K)$ is contained in a closed homogeneous half-space of $E^{\prime}$ (i.e., a set of the form $\left\{x^{\prime}:\left\langle x_{0}, x^{\prime}\right\rangle \leqq 0\right\}$ for some $\left.x_{0} \in E\right)$.

(c) $0^{+} K=\{0\}$ if and only if $\operatorname{bar}(K)$ is dense in $E^{\prime}$.

(c') $0^{+} K=\{0\}$ and $K$ is unbounded if and only if bar $(K)$ is a proper dense subset of $E^{\prime}$. In this case, bar $(K)$ has empty interior.

This result together with the Bishop-Phelps theorem on supporting functionals and James criterion for weak compactness yield immediately the following correspondence between properties of $K$ and properties of $\partial \delta_{K}(M)$ :

3.1.2. THEOREM. In addition to the hypotheses of the preceding theorem, assume that $E$ is complete. Then

(a) $\partial \delta_{K}(M)=E^{\prime}$ if and only if $K$ is $\sigma\left(E, E^{\prime}\right)$-compact.

If in addition, either $K$ is a convex body or $E$ is a Banach space (with dual $\left.E^{\prime}\right)$, then the $\beta\left(E^{\prime}, E\right)$-closure of $\partial \delta_{K}(M)$ is convex and

(b) $0^{+} K \neq\{0\}$ if and only if $\partial \delta_{K}(M)$ is contained in a closed, homogeneous half-space of $E^{\prime}$.

(c) $0^{+} K=\{0\}$ if and only if $\partial \delta_{K}(M)$ is dense in $E^{\prime}$. If nonempty, the interior (relative to any topology compatible with the 
linear structure of $\left.E^{\prime}\right)$ of $\beta\left(E^{\prime}, E\right)$-cl $[\mathrm{bar}(K)]$ is convex.

The hypothesis following (a) appears to be essential for (c), since (i) in any incomplete normed linear space there exists a bounded, closed, convex set $K$ with $\partial \delta_{K}(M)$ not dense in $E^{\prime}$ [5, Theorem 3], and (ii) there exists a closed, convex set with empty interior (in a complete, metrizable, topological vector space) with no supporting functionals $[23 ; 12$, page 166].

We now take $E$ to be the dual $X^{\prime}$ of a Banach space $X$ and take $E^{\prime}$ to be the "original" space $X$; then we are able to replace bar $(K)$ by $\partial \delta_{K}(M)$ in the assertions of Theorem 3.1.1 and thus obtain a precise generalization of de Andrade's Theorem A [2]:

3.1.3. THEOREM. Let $K$ be a $\sigma\left(X^{\prime}, X\right)$-closed ("weak*-closed"), nonempty convex set in the dual $X^{\prime}$ of a Banach space $X$. Let $M=\mathrm{bd}(K)$ and $\partial \delta_{K}(M)$ be the subset of $X$ containing points which generate supporting functionals of $K$. Then $\mathrm{cl}\left[\partial \delta_{K}(M)\right]$ is convex and

(a) $K$ is bounded if and only if $\partial \delta_{K}(M)=X$.

(b) $0^{+} K \neq\{0\}$ if and only if $\partial \delta_{K}(M)$ is contained in a closed, homogeneous half-space of $X$.

(c) $K$ is unbounded and $0^{+} K=\{0\}$ if and only if $\partial \delta_{K}(M)$ is dense in $X$ but is not equal to $X$. In this case, int $\left[\partial \delta_{K}(M)\right]=\varnothing$.

3.1.4. Corollary. If $K$ is a closed, convex subset of a reflexive Banach space E, then conclusions (a) to (c) of Theorem 3.1.3 hold with $X$ replaced by $E^{\prime}\left(\partial \delta_{K}(M) \subseteq E^{\prime}\right)$.

As the next theorem shows, the validity of conclusion (c) depends essentially on the reflexivity of the space in question.

3.1.5. THeorem. (a) Suppose $E$ is a quasi-complete [7, Chap. III, $\S 2$, No. 5], locally convex Hausdorff topological vector space with dual $E^{\prime}$. Then $\partial \delta_{K}(M)=E^{\prime}$ for every nonempty, bounded, closed, convex set $K \subseteq E$ if and only if $E$ is semireflexive.

(b) Suppose $K$ is a closed, convex body in a Banach space $E$. Then $\partial \delta_{K}(M)=E^{\prime}$ if and only if $E$ is reflexive and $K$ is bounded.

\section{Proofs of theorems of $\S 3$.}

4.1. On Theorem 3.1.1. Conclusion (a) is well-known; cf. [7, Chap. IV, $\S 2$, No. 4]. As pointed out above, $0^{+} K={ }^{\circ}[\operatorname{bar}(K)]$. By the bipolar theorem [7, Chap. IV, $\S 1$, No. 3; 12, page 68], $\left(0^{+} K\right)^{\circ}=$ ${ }^{\circ}[\operatorname{bar}(K)]^{\circ}$ is the $\sigma\left(E^{\prime}, E\right)$-closure of the convex set bar $(K)$, and is 
also (by convexity) the closure of bar $(K)$ in any topology compatible with the duality [7, Chap. IV, §2, No. 3]. Conclusions (b) and (c) follow immediately.

The first part of $\left(c^{\prime}\right)$ is obvious from (a) and (c). If any convex set $B$ (in particular, bar $(K)$ ) has nonempty interior, then its interior, int $(B)$, is equal to the interior of its closure, int $[\mathrm{cl}(B)][12$, page 59]. (In particular, if $\operatorname{int}[\operatorname{bar}(K)] \neq \varnothing$, then $\operatorname{int}[\operatorname{cl}(\operatorname{bar}(K))] \subseteq$ bar $(K)$; this is de Andrade's Lemma 2 [2] and Lemma 3 [1].) Thus if $\operatorname{cl}[\operatorname{bar}(K)]=E^{\prime}$ and $\operatorname{int}[\operatorname{bar}(K)] \neq \varnothing$, then $\operatorname{int}[\operatorname{bar}(K)]=E^{\prime}=$ bar $(K)$, so $K$ is bounded. This completes the demonstration of $\left(\mathbf{c}^{\prime}\right)$.

4.2. On Theorem 3.1.2. If $K$ is $\sigma\left(E, E^{\prime}\right)$-compact, then every continuous linear functional assumes its maximum on $K$ and thus is a supporting functional; therefore, $\partial \delta_{K}(M)=E^{\prime}$. Conversely, James [15; 14; cf. 10, Ch. $1 ; 12$, page 157] has shown that if every continuous linear functional assumes its maximum on a closed, convex set $K$, then $K$ is weakly compact. This completes the proof of (a). (This remains valid if $E$ is only quasi-complete.)

To prove (b) and (c), we use the following facts; Proposition 4.2.1 is a corollary of Theorem 3.1.1, while Proposition 4.2.2 follows from the Bishop-Phelps theorem. We use the notation of Theorem 3.1.1.

4.2.1. Proposition. (a) If $0^{+} K \neq\{0\}$, then $\partial \delta_{K}(M)$ is contained in a half-space of $E^{\prime}$.

(b) If $\partial \delta_{K}(M)$ is dense in $E^{\prime}$, then $0^{+} K=\{0\}$.

(c) If $\partial \delta_{K}(M)$ is dense in $E^{\prime}$ and has a nonempty interior, then $K$ is bounded.

4.2.2. Proposition. Suppose that $E$ is complete and either $K$ has nonempty interior or $E$ is normable. Then the polar of $\partial \delta_{K}(M)$ is the recession cone of $K$ :

$$
{ }^{\circ}\left[\partial \delta_{K}(M)\right]=0^{+} K \text {. }
$$

Proof. Under these hypotheses, a theorem of Bishop and Phelps [5, Theorem 2; 24, Corollary $1 ; 12$, page 165] asserts that $\partial \delta_{K}(M)$ is $\beta\left(E^{\prime}, E\right)$-dense in bar $(K)$. Thus

$$
\begin{aligned}
\partial \delta_{K}(M) & \cong \operatorname{bar}(K) \\
& \cong \beta\left(E^{\prime}, E\right) \text {-closure of } \partial \delta_{K}(M) \quad(\text { Bishop-Phelps }) \\
& \left.\cong \sigma\left(E^{\prime}, E\right) \text {-closure of } \partial \delta_{K}(M) \quad \text { (since } \beta\left(E^{\prime}, E\right) \supseteqq \sigma\left(E^{\prime}, E\right)\right) \\
& \cong\left({ }^{\circ}\left[\partial \delta_{K}(M)\right]\right)^{\circ} \quad \text { (bipolar theorem). }
\end{aligned}
$$


Thus ${ }^{\circ}\left[\partial \delta_{K}(M)\right]={ }^{\circ}[\operatorname{bar}(K)]=0^{+} K$.

Conclusions (b) and (c) of Theorem 3.1.2 now are obvious.

The $\beta\left(E^{\prime}, E\right)$-closure of $\partial \delta_{K}(M)$ is convex since, by the BishopPhelps theorem, it coincides with the $\beta\left(E^{\prime}, E\right)$-closure of the convex set bar $(K)$. The last assertion of 3.1.2 follows from the fact that in any topological vector space, the interior of a convex set is convex [6, Chap. II, $\S 2$, No. 6].

4.3. On Theorem 3.1.3. The Bishop-Phelps theorem which was used in the proof of Lemma 4.2.2 has a dual version which asserts that if $K$ is a weak*-closed, convex subset of the dual $X^{\prime}$ of a Banach space $X$, then the set of points in $X$ which generate supporting functionals of $K$ is norm-dense in the set of points in $X$ which generate functionals bounded above on $K$ [25]. Thus Theorem 3.1.3 can be proved just as Theorem 3.1.2 was proved. (In comparing Theorem 3.1.3 with Theorem 3.1.5, note that $X^{\prime}$ with the $\sigma\left(X^{\prime}, X\right)$ topology is semireflexive.)

4.4. On Theorem 3.1.5. Conclusion (a) is a consequence of James theorem on weak compactness (see Theorem 3.1.2(a)) and well-known characterizations of semireflexive spaces. Cf. Köthe $[20, \S 24,4$ (3)] and Klee [19], where it is shown, in fact, that if $E$ is not semireflexive, then given any nonzero $x^{\prime} \in E^{\prime}$, there exists a closed, bounded, absolutely convex set $K$ such that $x^{\prime}$ is not a supporting functional of $K$.

To prove (b), it suffices to show that if $\partial \delta_{K}(M)=E^{\prime}$ for a convex body $K$ in a Banach space $E$, then $E$ is reflexive. But James criterion again implies that $K$ is weakly compact. By translation, since $\operatorname{int}(K) \neq \varnothing$, so is the unit ball of $E$, and hence $E$ is reflexive. Cf. James [13].

5. Further discussion of int $[\operatorname{bar}(K)]$ and the case $0^{+} K \neq\{0\}$.

5.1. The analysis of the case in which $M=\mathrm{bd}(K)$ bounds a half-line - i.e., $0^{+} K \neq\{0\}$ - can be carried further than was done above or by de Andrade, for there is a relationship between the interior of $\mathrm{cl}[\operatorname{bar}(K)]$ (or $\mathrm{cl}\left[\partial \delta_{K}(M)\right]$ ) and the size of $0^{+} K$.

We recall the definition of a base of a cone $C$ in a linear space $E[12, \S 5 \mathrm{C}]$ : This is a convex subset $B \subseteq C$ with the property that for every nonzero $x \in C$, there is a unique $\lambda>0$ such that $\lambda x \in B$. A set $B$ is a base of $C$ if and only if there exists a linear functional $\phi$ on $E$ which is strictly positive on $C \backslash\{0\}$ and such that $B=$ $\{x \in C: \phi(x)=1\}$.

Theorem 5.1.1 lists necessary and sufficient conditions, in terms 
of the "size" of a base of $0^{+} K$, for cl [bar $\left.(K)\right]$ to have a nonempty interior. The proof uses known general results (due primarily to Moreau and to Rockafellar) on the relation between the interior of a cone and the base of its polar cone, as well as the polarities discussed in $\S \S 2$ and 4 :

$$
0^{+} K={ }^{\circ} \operatorname{bar}(K)={ }^{\circ} \mathrm{cl}[\operatorname{bar}(K)]
$$

and

$$
\left(0^{+} K\right)^{\circ}=\operatorname{cl}[\operatorname{bar}(K)] .
$$

(As before, we work with a dual pair $\left(E, E^{\prime}\right)$ with topologies compatible with the duality, and cl denotes the closure with respect to such a topology; for a convex set such as bar $(K)$, it is independent of the particular compatible topology used.) Proofs are given in $\S 6$.

5.1.1. THEOREM. Let $K$ be a closed, convex subset of $E$ with $0^{+} K \neq\{0\}$. Then

(a) The following are equivalent:

(i) $\operatorname{cor}[\mathrm{cl}[\mathrm{bar}(K)]] \neq \varnothing$;

(ii) $\beta\left(E^{\prime}, E\right)$-int $[\mathrm{cl}[\operatorname{bar}(K)]] \neq \varnothing$;

(iii) $0^{+} K$ has a bounded base.

(b) The following are equivalent;

(i ) int $[\mathrm{cl}[\operatorname{bar}(K)]] \neq \varnothing$;

(ii) $0^{+} K$ has a $\sigma\left(E, E^{\prime}\right)$-compact base;

(iii) $0^{+} K$ is locally compact.

(c) The following are equivalent:

(i ) $\sigma\left(E^{\prime}, E\right)$-int $[\mathrm{cl}[\operatorname{bar}(K)]] \neq \varnothing$;

(ii) $0^{+} K$ is finite dimensional and has a bounded base.

5.1.2. Corollary. Suppose $E$ is a semireflexive, locally convex topological vector space with topological dual $E^{\prime}$, and $K$ is a closed, convex subset of $E$, with $0^{+} K \neq\{0\}$. Then the following are equivalent:

(i) $\operatorname{cor}[\mathrm{cl}[\operatorname{bar}(K)]] \neq \varnothing$;

(ii) $\beta\left(E^{\prime}, E\right)$-int $[\mathrm{cl}[\operatorname{bar}(K)]] \neq \varnothing$;

(iii) $0^{+} K$ has a bounded (and hence weakly compact) base. $I f$, in addition, $E$ is complete and either $K$ has nonempty interior or $E$ is normable, then $\mathrm{cl}\left[\partial \delta_{K}(M)\right]=\mathrm{cl}[\operatorname{bar}(K)]$, and so "bar $(K)$ " may be replaced by " $\partial \delta_{K}(M)$ " in (i) and (ii).

As in Theorem 3.1.3 and Corollary 3.1.4, this result is immediately applicable to weak*-closed, convex subsets of the dual of a Banach space and to closed, convex subsets of a reflexive Banach space. 
If the interior of bar $(K)$ is nonempty, then int [cl [bar $(K)]]=$ int [bar $(K)]$. The following example shows, however, that 5.1.1 and 5.1.2 are not true if in them we replace the interior of the closure of bar $(K)$ in (i) or (ii) by the interior of bar $(K)$, because the latter interior may be empty.

5.1.3. ExAMPLE. Let $K_{0}$ be an unbounded, closed, convex subset of a Hilbert space $H_{0}$ with $0^{+} K_{0}=\{0\}$ (see, e.g., de Andrade [2]). By Theorem 3.1.1, bar $\left(K_{0}\right)$ is dense in $H_{0}$ and has an empty interior. Let $H$ be the Hilbert space direct sum $H_{0} \oplus \boldsymbol{R}$ of ordered pairs $(x, \lambda)$, with $x \in H_{0}, \lambda \in \boldsymbol{R}$; we identify the dual of $H$ with $H$. Define

$$
K=\left\{(x, \lambda) \in H: x \in K_{0}, \lambda \geqq 0\right\} .
$$

Then $0^{+} K=\{(0, \lambda) \in H: 0 \leqq \lambda \in R\}, \quad \operatorname{bar}(K)=\left\{(v, \mu) \in H: v \in \operatorname{bar}\left(K_{0}\right)\right\}$, $0 \geqq \mu \in \boldsymbol{R}$, and $\mathrm{cl}[\operatorname{bar}(K)]=\left(0^{+} K\right)^{\circ}=\left\{(v, \mu) \in H: v \in H_{0}, \mu \leqq 0\right\}$. Thus, although $0^{+} K$ is finite-dimensional and has a bounded base, int [bar $\left.(K)\right]$ is empty.

5.2. The following theorem describes the strong interior of bar $(K)$ directly in terms of the intersection of $K$ with halfspaces. It is proved in the same way as Theorem 5.1.1 and, in fact, is a generalization of Theorem 5.1.1(a), since the latter can be obtained by applying Theorem 5.2.1 to $0^{+} K$ in place of $K$ and using bar $\left(0^{+} K\right)=$ $\left(0^{+} K\right)^{\circ}=\mathrm{cl}[\mathrm{bar}(K)]$ (in any topology compatible with the duality). In a semireflexive space, it asserts that $v^{\prime} \in$ int [bar $\left.(K)\right]$ if and only if the intersection of $K$ with any halfspace $\left\{x:\left\langle x, v^{\prime}\right\rangle \geqq \alpha\right\}$ is bounded. The corollary is a generalization of de Andrade's condition (d) [2] for $K$ to be a "pseudograph" (cf. [30, Lemma 4]).

It is convenient to use the following notation: If $v^{\prime} \in E$, we denote by $\left[v^{\prime} \geqq \alpha\right]$ the halfspace

$$
\left[v^{\prime} \geqq \alpha\right]=\left\{x \in E:\left\langle x, v^{\prime}\right\rangle \geqq \alpha\right\} ;
$$

$\left[v^{\prime}>\alpha\right],\left[v^{\prime}=\alpha\right]$, etc., have analogous meanings.

5.2.1. THEOREM. Let $K$ be a nonempty, closed, convex subset of $E$. Suppose $0 \neq v^{\prime} \in E^{\prime}$. Then the following are equivalent:

(i ) $v^{\prime} \in \beta\left(E^{\prime}, E\right)$-int [bar $\left.(K)\right]$;

(ii) $v^{\prime} \in \operatorname{cor}[\operatorname{bar}(K)]$;

(iii) For all real $\alpha, K \cap\left[v^{\prime} \geqq \alpha\right]$ is bounded;

(iv) There exists a real $\beta$ such that

$K \cap\left[v^{\prime}>\beta\right]$ is nonempty and bounded.

If we weaken the condition (iv) to the condition that the intersection of $K$ with a nonsupporting hyperplane $\left[v^{\prime}=\alpha\right]$ is bounded 
and nonempty, then we can still conclude, in many cases, that $v^{\prime}$ (or $-v^{\prime}$ ) is an interior point of $\operatorname{bar}(K)$ (see Theorem 5.2.2 below). Some extra hypotheses are necessary, however, for if $K$ is a line or a cylinder, then the intersection of $K$ with certain hyperplanes $\left[v^{\prime}=\alpha\right]$ may be bounded, although $\pm v^{\prime} \notin$ bar $(K)$. We shall see that, at least in semireflexive spaces, if we rule out this cylindrical possibility, then the boundedness and nonemptiness of $K \cap\left[v^{\prime}=\alpha\right]$ for a nonsupporting hyperplane $\left[v^{\prime}=\alpha\right]$ implies $v^{\prime} \in \operatorname{int}[\operatorname{bar}(K)]$ or $-v^{\prime} \epsilon$ int [ $\operatorname{bar}(K)])$.

We shall say that a hyperplane properly supports $K$ if it is a supporting hyperplane which does not contain $K$. We then have the following extension of Theorem 5.2.1:

5.2.2. THEOREM. With the hypotheses of Theorem 5.2.1, each of the conditions (i) to (iv) of that Theorem implies each of the following equivalent conditions:

(v) There exists a real $\alpha$ such that the hyperplane $\left[v^{\prime}=\alpha\right]$ does not properly support $K$ and $K \cap\left[v^{\prime}=\alpha\right]$ is nonempty and bounded.

(vi) There exists a real $\alpha$ such that $\left[v^{\prime}=\alpha\right]$ does not properly support $K$ and $M \cap\left[v^{\prime}=\alpha\right]$ is nonempty and bounded.

Suppose, in addition, that $E$ is semireflexive (with dual $E^{\prime}$ ), and $0^{+} K$ is not a one-dimensional linear subspace of $E$. If some nonzero $v^{\prime} \in E^{\prime}$ satisfies one of the conditions (v) or (vi), then either $v^{\prime}$ or $-v^{\prime}$ satisfies each of the conditions (i) to (iv) of Theorem 5.2.1.

This theorem will be proved with the help of the following proposition, which completely describes the recession cone of $K$ in the case that $K \cap\left[v^{\prime}=\alpha\right]$ is compact (in the weak topology of a semireflexive space, this is equivalent to $K \cap\left[v^{\prime}=\alpha\right]$ being bounded), and which indicates the role played by $0^{+} K$ being a one-dimensional subspace.

5.2.3. Proposition. Let $K$ be a closed, convex subset of $E$. Suppose there exists a nonzero $v^{\prime} \in E^{\prime}$ and a real $\alpha_{0}$ such that the hyperplane $\left[v^{\prime}=\alpha_{0}\right]$ does not properly support $K$ and $K \cap\left[v^{\prime}=\alpha_{0}\right]$ is nonempty and compact. Then $K \cap\left[v^{\prime}=\alpha\right]$ is compact for every real $\alpha$ and

(a) $v^{\prime}$ is bounded on $K$ if and only if $K$ is compact.

(b) $v^{\prime}$ is not bounded above on $K$ if and only if $\left(0^{+} K\right) \cap$ $\left[v^{\prime}=1\right] \neq \varnothing$. In this case, $\left(0^{+} K\right) \cap\left[v^{\prime}=1\right]$ is compact and is a base for the cone $\left(0^{+} K\right) \cap\left[v^{\prime} \geqq 0\right]$.

(c) The following are equivalent:

(i ) $v^{\prime}$ is unbounded above and unbounded below on $K$; 
(ii) $\left(0^{+} K\right) \cap\left[v^{\prime}=1\right] \neq \varnothing$ and $\left(0^{+} K\right) \cap\left[v^{\prime}=-1\right] \neq \varnothing$;

(iii) $0^{+} K$ is a one-dimensional subspace of $E$.

If any one of these conditions (i)-(iii) holds, $K$ is "cylindrical" with cross sections parallel to $K \cap\left[v^{\prime}=0\right]$ and generators parallel to $0^{+} K$ : Given a nonzero $h \in 0^{+} K$, each $x \in K$ has a unique representation $x=x_{0}+\alpha h$ for $x_{0} \in K \cap\left[v^{\prime}=0\right]$ and $\alpha \in R$. Furthermore, $\operatorname{bar}(K)$ is the hyperplane which annihilates $0^{+} K$ :

$$
\operatorname{bar}(K)=\left\{x^{\prime} \in E^{\prime}:\left\langle x, x^{\prime}\right\rangle=0 \text { for all } x \in 0^{+} K\right\} .
$$

The following corollary follows from the preceding results together with special facts about the subdifferentials of convex functions in Banach spaces.

5.2.4. Corollary. Suppose $K, X$, and $X^{\prime}$ satisfy the hypotheses of Theorem 3.1.3, so that $\partial \delta_{K}(M) \subseteq \operatorname{bar}(K) \subseteq X$. Then int $[\operatorname{bar}(K)]=$ int $\left[\partial \delta_{K}(M)\right]$, this is a convex set, and the following are equivalent for any nonzero $v \in X$ :

(i) $v \in \operatorname{int}\left[\partial \delta_{K}(M)\right]$;

(ii) For some real $\alpha, K \cap[v \geqq \alpha]$ is nonempty and bounded. If (i) holds and $-v \notin$ bar $(K)$, then $\left(0^{+} K\right) \cap[v=-1]$ is a bounded base of $0^{+} K$. Furthermore, (i) and (ii) imply that for all real $\beta$

(iii) $K \cap\left[v^{\prime}=\beta\right]$ is bounded.

On the other hand, if (iii) is satisfied for some real $\beta$ such that the hyperplane $\left[v^{\prime}=\beta\right]$ does not properly support $K$, and if $0^{+} K$ is not a one-dimensional subspace, then either $v$ or $-v$ satisfies (i) and (ii).

\section{Proofs of results of $\S 5$.}

6.1. On Theorem 5.1.1. On (a): For any closed, convex set, the core is the same as the strong interior $[29 ; 21, \S 8 \mathrm{e}]$; thus (i) $\Leftrightarrow$ (ii). The equivalence (ii) $\Leftrightarrow$ (iii) follows from the polarity of bar $(K)$ and $0^{+} K$ [29, Corollary 7F; 16, Theorem 3.8.4]. On (b): The equivalence (ii) $\Leftrightarrow$ (iii) is valid for any closed, convex cone $[18 ; 12, \S 13 \mathrm{C}]$, while (i) $\Leftrightarrow$ (ii) follows again from the polarity of $\operatorname{bar}(K)$ and $0^{+} K[22$; 21, §8f, Exemple] and the fact that int [bar $(K)]=\operatorname{int}[\mathrm{cl}[\operatorname{bar}(K)]]$. On (c): Again this is a special case of a general result on polar cones and follows from a theorem of Moreau [21, $\S 8 \mathrm{~g}]$ on the equivalence of the weak continuity of a convex function and the finite dimensionality of the level sets of its convex conjugate.

6.2. Proof of Corollary. The first part of Corollary 5.1.2 is immediate, since the $\beta\left(E^{\prime}, E\right)$ topology on $E^{\prime}$ is compatible with the duality if $E$ is semireflexive. The last assertion of 5.1.2 follows 
from the fact that under its hypotheses, $\beta\left(E^{\prime}, E\right)$-cl $\left[\partial \delta_{K}(M)\right]=$ $\beta\left(E^{\prime}, E\right)$-cl $[$ bar $(K)]=\mathrm{cl}[$ bar $(K)]$ by the Bishop-Phelps theorem (cf. the proof of Proposition 4.2.2) and the semireflexivity of $E$.

6.3. On Theorem 5.2.1. The implications (i) $\Rightarrow$ (ii) and (iii) $\Rightarrow$ (iv) are obvious. If (ii) holds, we choose $\alpha_{0}<\sup \left\{\left\langle x, v^{\prime}\right\rangle: x \in K\right\}$ to conclude that $K \cap\left[v^{\prime} \geqq \alpha_{0}\right]$ is nonempty and bounded, by Theorem $4 \mathrm{C}$ of [29] applied to the indicator function of $\delta_{K}$ of $K$; by Corollary $4 \mathrm{D}$ of [29], $K \cap\left[v^{\prime} \geqq \alpha\right]$ is bounded for all $\alpha$. (Cf. the proof of Theorem 5.1.1 above and the proof of Corollary $7 \mathrm{~F}$ in [29].) Thus (ii) $\Rightarrow$ (iii). Suppose (iv) holds. Since $K \cap\left[v^{\prime}>\beta\right]=\mathrm{U}_{\alpha>\beta} K \cap\left[v^{\prime} \geqq \alpha\right]$, it follows that for all $\alpha$ sufficiently close to $\beta$ with $\alpha>\beta, K \cap$ $\left[v^{\prime} \geqq \alpha\right]$ is nonempty and bounded. We can choose such an $\alpha$ so that $\sup \left\{\left\langle x, v^{\prime}\right\rangle: x \in K\right\}>\alpha$, and then (i) (and (ii)) follow directly from Theorem $7 \mathrm{~A}(\mathrm{a})$ and Theorem $4 \mathrm{C}$ of [29].

6.4. On Proposition 5.2.3. We first prove the following general result, which will be applied to the weak topology on a semireflexive space $\mathrm{E}$ in proving Theorem 5.2.2.

6.4.1. Lemma. Suppose $K$ is a nonempty, closed, convex subset of $E$ and $0 \neq v^{\prime} \in E^{\prime}$. Then $K \cap\left[v^{\prime}=\alpha\right]$ is bounded (or compact) for every real $\alpha$ if and only if there exists a hyperplane $\left[v^{\prime}=\alpha_{0}\right]$ which does not properly support $K$ such that $K \cap\left[v^{\prime}=\alpha_{0}\right]$ is nonempty and bounded (or compact, respectively). If this is the case and if $v^{\prime}$ is bounded on $K$, then $K$ is bounded (or compact, respectively).

Proof. The first assertion, on the boundedness (or compactness) of the cross-sections $K \cap\left[v^{\prime}=\alpha\right]$ is easily verified. Suppose $v^{\prime}$ is bounded on $K$, so there exists $\beta>0$ such that $K \cong\left[-\beta<v^{\prime}<\beta\right]$. Suppose $K$ is unbounded; then there exists a convex neighborhood $U$ of 0 such that, for any increasing, divergent sequence $\left\{\lambda_{n}\right\}$ of positive reals, there exists $x_{n} \in K \backslash\left(\lambda_{n} U\right)$. Since $\left|\left\langle x_{n}, v^{\prime}\right\rangle\right|\langle\beta$ for all $n$, we may assume, by going to a subsequence if necessary, that $\left\{\left\langle x_{n}, v^{\prime}\right\rangle\right\}$ converges, say to $\beta_{1}$, with $\left|\beta_{1}\right| \leqq \beta$. Since $K \cap\left[v^{\prime}=\beta_{1}\right]$ is bounded, we may choose $x_{0} \in K$ with $\left\langle x_{0}, v^{\prime}\right\rangle=\beta_{0} \neq \beta_{1}$; let $\beta_{2}$ be a number strictly between $\beta_{0}$ and $\beta_{1}$. We may assume $\left\langle x_{n}, v^{\prime}\right\rangle$ lies between $\beta_{2}$ and $\beta_{1}$ for every $n$. Let

$$
\eta_{n}=\frac{\beta_{2}-\beta_{0}}{\left\langle x_{n}, v^{\prime}\right\rangle-\beta_{0}}=\frac{\beta_{0}-\beta_{2}}{\beta_{0}-\left\langle x_{n}, v^{\prime}\right\rangle} .
$$

Then $0<\eta_{n}<1$, so 


$$
\left(1-\eta_{n}\right) x_{0}+\eta_{n} x_{n} \in K \cap\left[v^{\prime}=\beta_{2}\right]
$$

for every $n \geqq 1$. Since $K \cap\left[v^{\prime}=\beta_{2}\right]$ is bounded, there exists $\lambda>0$ such that

$$
\eta_{n} x_{n}+\left(1-\eta_{n}\right) x_{0} \in \lambda U
$$

for every $n \geqq 1$; hence there exists $\mu>0$ such that $\eta_{n} x_{n} \in \mu U$ for all $n \geqq 1$. Now $\eta_{n}^{-1} \leqq\left(\beta+\left|\beta_{0}\right|\right) /\left|\beta_{0}-\beta_{1}\right|$, so

$$
x_{n} \in \mu\left(\frac{\beta+\left|\beta_{0}\right|}{\left|\beta_{0}-\beta_{1}\right|}\right) U
$$

for every $n \geqq 1$. This contradicts the unboundedness of $\left\{x_{n}\right\}$.

A similar argument shows that if $K \cap\left[v^{\prime}=\beta_{2}\right]$ is compact, then any net in $K$ contains a convergent subnet.

Proof of Proposition 5.2.3. Assertion (a) follows from Lemma 6.4.1. Suppose $v^{\prime}$ is not bounded above on $K$. Then there exists a positive number $k_{0}$ such that, for every integer $n \geqq 0$, there is an $x_{n} \in K$ with $\left\langle x_{n}, v^{\prime}\right\rangle \geqq n+k_{0}=n+\left\langle x_{n}, v^{\prime}\right\rangle$. For $n \geqq 1$, let $\eta_{n}=$ $1 /\left\langle x_{n}-x_{0}, v^{\prime}\right\rangle$ (so $0<\eta_{n} \leqq 1$ ) and $z_{n}=\left(1-\eta_{n}\right) x_{0}+\eta_{n} x_{n}$; then $z_{n} \in K \cap$ $\left[v^{\prime}=k_{0}+1\right]$. Because $K \cap\left[v^{\prime}=k_{0}+1\right]$ is compact (by Lemma 6.4.1) we can find subnets $\left\{w_{\hat{o}}\right\},\left\{\rho_{\hat{o}}\right\}$, and $\left\{y_{\hat{o}}\right\}$ of $\left\{z_{n}\right\},\left\{\eta_{n}\right\}$, and $\left\{x_{n}\right\}$, respectively, such that $w_{\hat{o}}=\left(1-\rho_{\hat{o}}\right) x_{0}+\rho_{\hat{o}} y_{\hat{o}}$, and $\left\{w_{\hat{o}}\right\}$ converges to some (nonzero) $h \in E$ with $\left\langle h, v^{\prime}\right\rangle=1+k_{0}$. Since $\rho_{\hat{o}} \rightarrow 0, \rho_{\hat{o}} y_{\hat{o}}$ converges to $h-x_{0}$ and therefore $h-x_{0} \in 0^{+} K \cap\left[v^{\prime}=1\right]$ [28, equation (2.2) and Theorem $2 \mathrm{~A}(\mathrm{e})]$. This proves (b) in one direction; the other direction is obvious. Suppose next that $v^{\prime}$ is not bounded above or below on $K$. Then (b) shows that there exist $h \in\left(0^{+} K\right) \cap\left[v^{\prime}=1\right]$ and $k \in\left(0^{+} K\right) \cap$ $\left[v^{\prime}=-1\right]$. If for some such $h$ and $k, h+k$ were not zero, then the two-dimensional cone with vertex at a point $x_{0} \in K \cap\left[v^{\prime}=0\right]$ and generated by $x_{0}+h$ and $x_{0}+k$ would be a subset of $K$ with unbounded intersection with $K \cap\left[v^{\prime}=1\right]$, in contradiction to the hypothesis and Lemma 6.4.1 (for all $\lambda \geqq 0, x_{0}+(1+\lambda) h+\lambda k \in K \cap\left[v^{\prime}=1\right]$ ). It follows that $0^{+} K$ is a one-dimensional subspace. We have thus shown that in (c), (i) $\Rightarrow$ (ii) $\Rightarrow$ (iii). If (iii) holds, then $0^{+} K \nsubseteq\left[v^{\prime}=0\right]$ since $K \cap\left[v^{\prime}=\alpha_{0}\right]$ is bounded. Hence there exists $h \in\left(0^{+} K\right) \cap\left[v^{\prime}=1\right]$, from which it follows that $v^{\prime}$ is not bounded above or below on $K$. Thus (iii) $\Rightarrow$ (i). The "cylindrical" structure of $K$ follows immediately. Consider now bar $(K)$; we know cl $[\operatorname{bar}(K)]=\left(0^{+} K\right)^{\circ}=\left(0^{+} K\right)^{\perp}=\left\{x^{\prime} \in E^{\prime}:\left\langle x, x^{\prime}\right\rangle=0\right.$ for all $\left.x \in 0^{+} K\right\}$ since $0^{+} K$ is a subspace. Choose $h \in 0^{+} K(h \neq 0)$ and $x^{\prime} \in \operatorname{cl}[\operatorname{bar}(K)]=\left(0^{+} K\right)^{\prime}$. For any $x \in K$, there exists $x_{0} \in K \cap\left[v^{\prime}=0\right]$ and $\alpha \in \boldsymbol{R}$ such that $x=x_{0}+\alpha h$. Then $\left\langle x, x^{\prime}\right\rangle=\left\langle x_{0}, x^{\prime}\right\rangle+\alpha\left\langle h, x^{\prime}\right\rangle=$ $\left\langle x_{0}, x^{\prime}\right\rangle$. Since $K \cap\left[v^{\prime}=0\right]$ is bounded, $x^{\prime}$ is bounded on $K \cap\left[v^{\prime}=0\right]$, 
and therefore $x^{\prime}$ is bounded on $K$. Thus $\operatorname{bar}(K)=\mathrm{cl}[\operatorname{bar}(K)]=$ $\left(0^{+} K\right)^{\perp}$.

6.4.2. Remarks. (a) If the subspace $\left[v^{\prime}=0\right]$ in Proposition 5.2.3 is semireflexive, then the conclusions (a) to (c) hold in the weak topology if $K \cap\left[v^{\prime}=\alpha_{0}\right]$ is assumed nonempty and bounded.

(b) It is easy to prove the following partial converse: Suppose $K$ is a closed, convex subset of a locally convex space $E$. If $0^{+} K$ is a one-dimensional subspace of $E$, then there exists a nonzero $v^{\prime}$ in the dual space $E^{\prime}$ of $E$ such that, for every real number $\alpha, K \cap$ $\left[v^{\prime}=\alpha\right]$ is nonempty and bounds no half-lines.

6.5. On Theorem 5.2.2. First we show that (v) and (vi) are equivalent. For any convex set $C$, if $b d(C)$ is bounded and nonempty, then $C$ is the convex hull of bd $(C)[3,1.7 .7(\mathrm{c})]$; thus $C$ is bounded if and only if bd $(C)$ is bounded and nonempty. Applying this to $K \cap\left[v^{\prime}=\alpha\right]$ and $M \cap\left[v^{\prime}=\alpha\right]$ in the hyperplane $\left[v^{\prime}=\alpha\right]$, and using the fact that $[\operatorname{cor}(K)] \cap\left[v^{\prime}=\alpha\right]$ is a subset of the core relative to $\left[v^{\prime}=\alpha\right]$ of $K \cap\left[v^{\prime}=\alpha\right]$, we see that (v) and (vi) are equivalent.

Clearly, (iv) of 5.2.1 implies both (v) and (vi). To prove the converse, we establish the following general result (with only the usual assumptions on $E$ and $K$ ):

6.5.1. Lemma. Suppose $v^{\prime} \in \operatorname{bar}(K)$ satisfies (v) or (vi) of 5.2.2, and $0^{+} K \neq\{0\}$. Then $v^{\prime} \in \beta\left(E^{\prime}, E\right)$-int (bar $\left.(K)\right]$.

Proof. Since $v^{\prime} \in \operatorname{bar}(K)$, there exists $\gamma \in \boldsymbol{R}$ such that $K \subseteq$ $\left[v^{\prime} \leqq \gamma\right]$. Since $K \cap\left\lfloor v^{\prime}=\alpha\right]$ is bounded and $0^{+} K \neq\{0\}, K \nsubseteq\left[v^{\prime}=\alpha\right]$. Hence $\left[v^{\prime}=\alpha\right]$ intersects $K$ but does not support it, and hence $\alpha<\gamma$ and $K \cap\left[v^{\prime}>\alpha\right]$ is nonempty. From the fact that $K \cap\left[v^{\prime}=\alpha\right]$ is bounded, it follows that $0^{+} K \nsubseteq\left[v^{\prime}=0\right]$, so there exists $x_{0} \in 0^{+} K$ $\left(={ }^{\circ} \operatorname{bar}(K)\right)$ such that $\left\langle x_{0}, v^{\prime}\right\rangle=-1$. It is easy to verify that

$$
K \cap\left[v^{\prime}>\alpha\right] \subseteq\left(K \cap\left[v^{\prime}=\alpha\right]\right)+[\alpha-\gamma, 0) x_{0},
$$

where

$$
[\alpha-\gamma, 0) x_{0}=\left\{\lambda x_{0}: \alpha-\gamma \leqq \lambda<0\right\} .
$$

Since the sum of two bounded sets is bounded, $K \cap\left[v^{\prime}>\alpha\right]$ is bounded. This verifies the lemma, by 5.2.1(iv) and (i).

We now return to the proof of Theorem 5.2.2. Suppose that $E$ is semireflexive and $0^{+} K$ is not a one-dimensional subspace of $E$, and that $v^{\prime} \in E^{\prime}$ satisfies (v). By Proposition 5.2.3 (applied to $E$ with the $\sigma\left(E, E^{\prime}\right)$ topology, in which the closed, bounded sets are compact), 
either $v^{\prime} \in \operatorname{bar}(K)$ or $-v^{\prime} \in \operatorname{bar}(K)$. If both $v^{\prime} \in \operatorname{bar}(K)$ and $-v^{\prime} \in$ bar $(K)$, then, by 5.2.3(a), $K$ is bounded and weakly compact, $\operatorname{bar}(K)=E$, and trivally $v^{\prime} \in \operatorname{int}[\operatorname{bar}(K)]$. If only one of $v^{\prime}$ or $-v^{\prime}$ belongs to bar $(K)$, then by $5.2 .3(\mathrm{~b}), 0^{+} K \neq\{0\}$, so Lemma 6.5.1 implies $v^{\prime} \in \beta\left(E^{\prime}, E\right)$-int [bar $\left.(K)\right]$ or $-v^{\prime} \in \beta\left(E^{\prime}, E\right)$-int [bar $\left.(K)\right]$.

6.6. On Corollary 5.2.4. Since bar $(K)$ is the effective domain of the support function $\delta_{K}^{*}: X \rightarrow \boldsymbol{R}$,

$$
\delta_{K}^{*}(x)=\sup \left\{\left\langle x, x^{\prime}\right\rangle: x^{\prime} \in K\right\},
$$

and $\partial \delta_{K}(M)\left(=\partial \delta_{K}(K)\right)$ is the domain of the subgradient $\partial \delta_{K}^{*}: X \rightarrow$ (subsets of $X^{\prime}$ ) of $\delta_{K}^{*}$,

$$
\begin{aligned}
\partial \delta_{K}^{*}(x) & =\left\{x^{\prime}: \delta_{K}^{*}(y)-\delta_{K}^{*}(x) \geqq\left\langle y-x, x^{\prime}\right\rangle \text { for all } y \in X\right\} \\
& =\left\{x^{\prime}: x \in \partial \delta_{K}\left(x^{\prime}\right)\right\}
\end{aligned}
$$

the fact that int $[\operatorname{bar}(K)]=\operatorname{int}\left[\partial \delta_{K}(M)\right]$ follows from known facts about convex functions and their subgradients on Banach spaces (cf. [28, page $57 ; 8]$ ). Being the interior of a convex set, int $[\operatorname{bar}(K)]$ is convex. The rest of the corollary follows directly from the preceding results.

\section{REFERENCES}

1. Rubens Leão de Andrade, Spherical images of continuous convex surfaces of Hilbert spaces, Bol. Soc. Brasil. Mat., 4 (1973), 41-45.

2. Complete convex hypersurfaces of a Hilbert space, J. Differential Geometry, 10 (1975), 491-499.

3. Jacques Bair and René Fourneau, Etude Geometrique des Espaces Vectoriels-I'ne Introduction, Lecture Notes in Mathematics, 489, Springer-Verlag, New York, 1975.

4. Russell V. Benson, Euclidean Geometry and Convexity, McGraw-Hill, New York, 1966.

5. E. Bishop and R. R. Phelps, Support functionals of convex sets, Proc. Symposia in Pure Math., Amer. Math. Soc., 7 (Convexity) (1963), 27-35.

6. N. Bourbaki, Éléments de Mathematique, Livre V, Espaces Vectoriels Topologiques, Fasc. XV, Hermann, Paris, 1966.

7. - Éléments de Mathematique, Livre V, Espaces Vectoriels Topologiques, Fasc. XVIII, Hermann, Paris, 1964.

8. A. Brøndsted and R. T. Rockafellar, On the subdifferentiability of convex functions, Bull. Amer. Math. Soc., 16 (1965), 605-611.

9. M. P. do Carmo and H. B. Lawson, Spherical images of convex surfaces, Proc. Amer. Math. Soc., 31 (1972), 635-636.

10. Joseph Diestel, Geometry of Banach Spaces-Selected Topics, Lecture Notes in Mathematics, 485, Springer-Verlag, New York, 1975.

11. Richard B. Holmes, A Course on Optimization and Best Approximation, Lecture Notes in Mathematics, 257, Springer-Verlag, Berlin, 1972.

12. - Geometric Functional Analysis and Its Applications, Springer-Verlag, New York, 1975.

13. R. C. James, Characterizations of reflexivity, Studia Math., 23 (1964), 205-216. 
14. R. C. James, Reflexivity and the sup of linear functionals, Israel J. Math., 13 (1972), 289-300.

15. Weakly compact sets, Trans. Amer. Math. Soc., 113 (1964), 129-140.

16. Graham Jameson, Ordered Linear Spaces, Lecture Notes in Mathematics, 141, Springer-Verlag, Berlin, 1970.

17. J. L. Kelley, Isaac Namioka, et al., Linear Topological Spaces, Van Nostrand, Princeton, 1963.

18. V. L. Klee, Separation properties for convex cones, Proc. Amer. Math. Soc., 6 (1950), 313-318.

19. - Some characterizations of reflexivity, Rev. Ci. (Lima), 52 (1950), 15-23.

20. Gottfried Köthe, Topological Vector Spaces I, Springer-Verlag, New York, 1969.

21. J. J. Moreau, Functionelles Convexes, Seminaire sur les Équations aux Dérivées Partielles II, Collège de France, 1966-67.

22. - Sur la fonction polaire d'une fonction semi-continue supérieurement, C. R. Acad. Sci Paris, 258 (1964), 1128-1131.

23. N. Peck, Support points in locally convex spaces, Duke Math. J., 38 (1971), 271-278.

24. R. R. Phelps, Support cones and their generalizations, Proc. Symp. Pure Math., 7 (convexity) (1963), 393-401.

25. - Weak* support points of convex sets in $E^{*}$, Israel J. Math., 2 (1964), 177-182.

26. R. Tyrrell Rockafellar, Conjugate Duality and Optimization, CBMS Regional Conference Series in Applied Mathematics, 16, SIAM, Philadelphia, 1974.

27. - Convex Analysis, Princeton University Press, Princeton, 1970.

28. - Convex functions, monotone operators and variational inequalities, in Theory and Application of Monotone Operators, Aldo Ghizetti, ed., 1968, Nato Advanced Study Institute, Gubbio, 1969.

29. Level sets and continuity of conjugate convex functions, Trans. Amer. Math. Soc., 123 (1966), 46-63.

30. H. Wu, The spherical images of convex hypersurfaces, J. Differential Geometry, 9 (1974), 279-290.

31. - A structure theorem for complete noncompact hypersurfaces of nonnegative curvature, Bull. Amer. Math. Soc., 77 (1971), 1070-1071.

Received April 4, 1977.

UNIVERSITY OF ARIZONA

Tucson, AZ 85721 



\section{PACIFIC JOURNAL OF MATHEMATICS}

EDITORS

RICHARD ARENS (Managing Editor)

University of California

Los Angeles, California 90024

C. W. Curtis

University of Oregon

Eugene, OR 97403

C. C. MOORE

J. DUGUNDJI

Department of Mathematics University of Southern Californa Los Angeles, California 90007

R. Finn AND J. Milgram Stanford University Stanford, California 94305

University of California

Berkeley, CA 94720

\section{ASSOCIATE EDITORS}

E. F. BeCKENBACH

B. H. NeUMANN

F. WOLF

K. YosHida

\section{SUPPORTING INSTITUTIONS}

UNIVERSITY OF BRITISH COLUMBIA UNIVERSITY OF SOUTHERN CALIFORNIA CALIFORNIA INSTITUTE OF TECHNOLOGY UNIVERSITY OF CALIFORNIA MONTANA STATE UNIVERSITY UNIVERSITY OF NEVADA, RENO STANFORD UNIVERSITY UNIVERSITY OF TOKYO UNIVERSITY OF UTAH NEW MEXICO STATE UNIVERSITY WASHINGTON STATE UNIVERSITY OREGON STATE UNIVERSITY UNIVERSITY OF WASHINGTON UNIVERSITY OF OREGON OSAKA UNIVERSITY 


\section{Pacific Journal of Mathematics \\ Vol. 73, No. 1 \\ March, 1977}

Thomas Robert Berger, Hall-Higman type theorems. $V \ldots \ldots \ldots \ldots \ldots \ldots \ldots$

Frank Peter Anthony Cass and Billy E. Rhoades, Mercerian theorems via

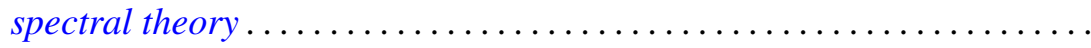

Morris Leroy Eaton and Michael David Perlman, Generating $\mathrm{O}(n)$ with

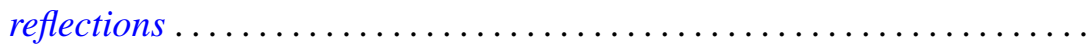

Frank John Forelli, Jr., A necessary condition on the extreme points of a

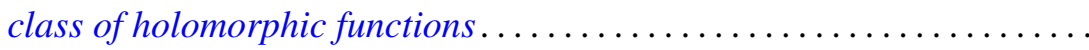

Melvin F. Janowitz, Complemented congruences on complemented

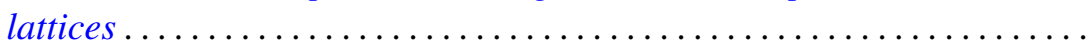

Maria M. Klawe, Semidirect product of semigroups in relation to amenability, cancellation properties, and strong $F \phi$ lner conditions....

Theodore Willis Laetsch, Normal cones, barrier cones, and the "spherical image" of convex surfaces in locally convex spaces ................

Chao-Chu Liang, Involutions fixing codimension two knots.............

Joyce Longman, On generalizations of alternative algebras .............

Giancarlo Mauceri, Square integrable representations and the Fourier

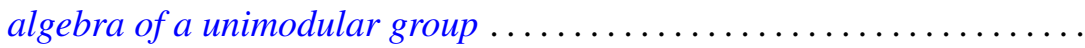

J. Marshall Osborn, Lie algebras with descending chain condition...

John Robert Quine, Jr., Tangent winding numbers and branched

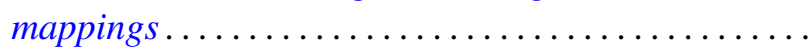

Louis Jackson Ratliff, Jr. and David Eugene Rush, Notes on ideal covers and associated primes .

H. B. Reiter and N. Stavrakas, On the compactness of the hyperspace of faces.

Walter Roth, A general Rudin-Carlson theorem in Banach-spaces ..

Mark Andrew Smith, Products of Banach spaces that are uniformly rotund in every direction.

Roger R. Smith, The R-Borel structure on a Choquet simplex ...

Gerald Stoller, The convergence-preserving rearrangements of real infinite series. ...

Graham H. Toomer, Generalized homotopy excision theorems modulo a Serre class of nilpotent groups...

Norris Freeman Weaver, Dehn's construction and the Poincaré conjecture....

Steven Howard Weintraub, Topological realization of equivariant intersection forms... 